\title{
A TWO-DIMENSIONAL FINITE ELEMENT MODEL OF THE GRAIN BOUNDARY BASED ON THERMO-MECHANICAL STRAIN GRADIENT PLASTICITY
}

\author{
Yooseob Song, George Z. Voyiadjis \\ Louisiana State University, Department of Civil and Environmental Engineering, Baton Rouge, LA, USA \\ e-mail: voyiadjis@eng.lsu.edu
}

\begin{abstract}
In this work, a two-dimensional finite element model for the grain boundary flow rule is developed based on the thermo-mechanical gradient-enhanced plasticity theory. The proposed model is temperature-dependent. A special attention is given to physical and micromechanical nature of dislocation interactions in combination with thermal activation on stored and dissipated energy. Thermodynamic conjugate microforces are decomposed into energetic and dissipative components. Correspondingly, two different grain boundary material length scales are present in the proposed model. Finally, numerical examples are solved in order to explore characteristics of the proposed grain boundary flow rule.
\end{abstract}

Keywords: strain gradient plasticity, grain boundary, energetic, dissipative, 2D FEM

\section{Introduction}

It is well known that the free surface may act as a source of defect development and its propagation towards the grain inside, whereas the grain boundaries block this dislocation movement, consequently, give rise to the strain gradients to accommodate geometrically necessary dislocations (Hirth and Lothe, 1982). In addition, the grain boundaries can be a source of dislocations through transmission of plastic slip to the neighboring grains (Clark et al., 1992). Besides these physical manifestationa, from the mathematical viewpoint, nonstandard boundary conditions are necessary at the external boundary of a region for the well-posed governing equations in the implementation of higher order strain gradient plasticity models. Therefore, careful modeling of the grain boundary is important in the continued development of higher order strain gradient plasticity models.

The experimental observations on slip transmission motivate one to assume that the effect of surface/interfacial energy and the global nonlocal energy residual should be non-vanishing. Examples can be found from the in-situ TEM direct observations, see e.g., Lee et al. (1989), or using the geometrically necessary dislocation (GND) concept in the description of observations in bicrystallines, e.g., Sun et al. (2000) and nanoindentation tests close to the grain boundary, e.g., Soer et al. (2005). This results in a new type of the boundary condition, in the context of strain gradient plasticity incorporating the interfacial energy, accounting for the surface resistance to slip transfer due to grain boundary misalignment, see e.g., Aifantis and Willis (2005), Cermelli and Gurtin (2002), Fredriksson and Gudmundson (2007), Gudmundson (2004), Gurtin (2008).

Voyiadjis and co-workers (Voyiadjis et al., 2014, 2017; Voyiadjis and Song, 2017) developed thermodynamically consistent and coupled thermo-mechanical strain gradient plasticity models incorporating the flow rules for both the grain interior and grain boundary to study characteristics of nano/micro-scale metallic materials. In those three works, the finite element analysis 
was implemented via a one-dimensional model. As is well known, there is bound to be a fundamental difference between one-dimensional finite element implementation and two-dimensional one. For example, in the one-dimensional case, some special complications, e.g. the resonance between the physical scale and the mesh scale, cannot be considered during simulation. In terms of dimensional extension, there were simple modifications from one-dimensional finite element implementation for the strain gradient plasticity model to the two-dimensional one in Voyiadjis and Song (2017) and Song and Voyiadjis (2018). However, in Voyiadjis and Song (2017), the grain boundary modeling and the effects of temperature and its gradient were not considered, but just addressed the effect of the mechanical component of thermodynamic microforces in terms of the stress jump phenomenon. In addition, in Song and Voyiadjis (2018), only two null boundary conditions, i.e. microscopically free and hard boundary conditions, were considered at the grain boundary to describe the dislocation movement and the plastic flow at the grain boundary areas. In the current work, two-dimensional numerical simulation, in the context of the small deformation framework, is developed incorporating temperature and rate dependent flow rules for the grain interior and grain boundary. The proposed model is applied to the simple shear problem in order to examine the characteristics of the proposed model.

\section{Thermodynamically consistent strain gradient plasticity model for grain interior}

In this work, tensors are denoted by the subscripts $i, j, k, l, m$, and $n$. The superscripts $e, p$, int, ext, en, dis and etc. imply specific quantities such as elastic state, plastic state, internal, external, energetic, dissipative and etc., respectively. Also, the superimposed dot represents derivative with respect to time, and the indices after a comma represent partial derivatives.

\subsection{Principle of virtual power (grain interior)}

The internal power $\mathcal{P}^{i n t}$ is presented with a combination of three energy contributions, i.e. the macro-, micro- and thermal-energy contributions, in an arbitrary region $\Omega_{0}$ as follows

$$
\mathcal{P}^{\text {int }}=\int_{\Omega_{0}}(\underbrace{\sigma_{i j} \dot{\varepsilon}_{i j}^{e}}_{\text {Macro }}+\underbrace{x \dot{e}^{p}+Q_{i} \dot{e}_{, i}^{p}}_{\text {Micro }}+\underbrace{\mathcal{A} \dot{\mathcal{T}}+\mathcal{B}_{i} \dot{\mathcal{T}}_{, i}}_{\text {Thermal }}) d V
$$

where $\varepsilon_{i j}^{e}$ is the elastic part of the strain tensor, $e^{p}$ is the accumulated plastic strain, $x$ and $Q_{i}$ are the thermodynamic microforces conjugate respectively to $\dot{e}^{p}$ and $\dot{e}_{, i}^{p}, \mathcal{A}$ and $\mathcal{B}_{i}$ are the micromorphic scalar and vector generalized stresses conjugate to the temperature rate $\dot{\mathcal{T}}$ and the gradient of the temperature rate $\dot{\mathcal{T}}_{, i}$ respectively, and $\sigma_{i j}$ is the Cauchy stress tensor.

The internal power $\mathcal{P}^{i n t}$ for $\Omega_{0}$ is equated with the external power $\mathcal{P}^{\text {ext }}$ expended by the macro and microtractions $\left(t_{i}, m\right)$ on the external surface $\partial \Omega_{0}$ and the body forces acting within $\Omega_{0}$ as follows

$$
\mathcal{P}^{e x t}=\int_{\Omega_{0}} \underbrace{b_{i} \dot{u}_{i}}_{\text {Macro }} d V+\int_{\partial \Omega_{0}}(\underbrace{t_{i} \dot{u}_{i}}_{\text {Macro }}+\underbrace{m \dot{e}^{p}}_{\text {Micro }}+\underbrace{a \dot{\mathcal{T}}}_{\text {Thermal }}) d S
$$

where $b_{i}$ is the generalized external body force conjugate to the macroscopic velocity $\dot{u}_{i}$. Furthermore, it is assumed for the external power to have the term of a conjugate to $\dot{\mathcal{T}}$ for the thermal effect.

By using the equation, $\mathcal{P}^{i n t}=\mathcal{P}^{e x t}$, in conjunction with the divergence theorem and factoring out the common terms, the balance equations for the macroscopic linear momentum, nonlocal 
microforce and generalized stresses $\mathcal{A}$ and $\mathcal{B}_{i}$ for the volume $\Omega_{0}$ can be obtained respectively as follows

$$
\sigma_{i j, j}+b_{i}=0 \quad \bar{\sigma}_{i j}=\left(x-Q_{k, k}\right) N_{i j} \quad \mathcal{B}_{i, i}-\mathcal{A}=0
$$

where $\bar{\sigma}_{i j}$ is the deviatoric part of $\sigma_{i j}$ with the Kronecker delta $\delta_{i j}\left(\bar{\sigma}_{i j}=\sigma_{i j}-\sigma_{k k} \delta_{i j} / 3\right)$.

On $\partial \Omega_{0}$, the balance equations for the local surface traction and the nonlocal microtraction are expressed with the outward unit normal vector to $\partial \Omega_{0}, n_{i}$, respectively, as

$$
t_{j}=\sigma_{i j} n_{i} \quad m=Q_{i} n_{i} \quad a=\mathcal{B}_{i} n_{i}
$$

\subsection{Second law of thermodynamics (grain interior)}

The second law of thermodynamics introduces a physical base to account for the GNDs distribution in the body. The following entropy production inequality can be obtained based on the basic statement of this law with the specific entropy $s$ and the micromorphic approach by Forest (2009)

$$
-\rho \dot{\mathcal{E}}+\rho \dot{\mathcal{T}} \mathcal{T}+\sigma_{i j} \dot{\varepsilon}_{i j}^{e}+x \dot{e}^{p}+Q_{i} \dot{e}_{, i}^{p}+\mathcal{A} \dot{\mathcal{T}}+\mathcal{B}_{i} \dot{\mathcal{T}}_{, i}-q_{i} \frac{\mathcal{T}_{, i}}{\dot{\mathcal{T}}} \geqslant 0
$$

The entropy production vector is assumed in this work to be equal to the thermal flux vector divided by temperature, as given in Coleman and Noll (1963).

\subsection{Energetic and dissipative thermodynamic microforces (grain interior)}

The Helmholtz free energy $\Psi$ (per unit volume) is obtained with the entropy $s$, internal energy $\mathcal{E}$ and temperature $\mathcal{T}$ describing the current state of the material as $\Psi=\mathcal{E}-\mathcal{T} s$. By using this equation along with Eq. (2.6), the Clausius-Duhem inequality is derived as follows

$$
\sigma_{i j} \dot{\varepsilon}_{i j}^{e}+x \dot{e}^{p}+Q_{i} \dot{e}_{, i}^{p}+\mathcal{A} \dot{\mathcal{T}}+\mathcal{B}_{i} \dot{\mathcal{T}}_{, i}-\rho \dot{\Psi}-\rho s \dot{\mathcal{T}}-q_{i} \frac{\mathcal{T}_{, i}}{\mathcal{T}} \geqslant 0
$$

For deriving the constitutive equations, the functional form of the Helmholtz free energy, $\Psi=\Psi\left(\varepsilon_{i j}^{e}, e^{p}, e_{, i}^{p}, \mathcal{T}, \mathcal{T}_{, i}\right)$, is put forward in this work. By taking time derivative of the Helmholtz free energy, $\dot{\Psi}$ is expressed as follows

$$
\dot{\Psi}=\frac{\partial \Psi}{\partial \varepsilon_{i j}^{e}} \dot{\varepsilon}_{i j}^{e}+\frac{\partial \Psi}{\partial e^{p}} \dot{e}^{p}+\frac{\partial \Psi}{\partial e_{, i}^{p}} \dot{e}_{, i}^{p}+\frac{\partial \Psi}{\partial \mathcal{T}} \dot{\mathcal{T}}+\frac{\partial \Psi}{\partial \mathcal{T}_{, i}} \dot{\mathcal{T}}_{, i}
$$

Substituting Eq. (2.7) into Eq. (2.6) and factoring the common terms out gives

$$
\begin{aligned}
& \left(\sigma_{i j}-\rho \frac{\partial \Psi}{\partial \varepsilon_{i j}^{e}}\right) \dot{\varepsilon}_{i j}^{e}+\left(x-\rho \frac{\partial \Psi}{\partial e^{p}}\right) \dot{e}^{p}+\left(Q_{i}-\rho \frac{\partial \Psi}{\partial e_{, i}^{p}}\right) \dot{e}_{, i}^{p} \\
& +\left(\mathcal{A}-\rho s-\rho \frac{\partial \Psi}{\partial \mathcal{T}}\right) \dot{\mathcal{T}}+\left(\mathcal{B}_{i}-\rho \frac{\partial \Psi}{\partial \mathcal{T}_{, i}}\right) \dot{\mathcal{T}}_{, i}-\frac{q_{i}}{\mathcal{T}} \mathcal{T}_{, i} \geqslant 0
\end{aligned}
$$

Meanwhile, the thermodynamic conjugate microforces $x, Q_{i}$ and $\mathcal{A}$ are assumed to be decomposed into the energetic and the dissipative elements as follows

$$
x=x^{e n}+x^{\text {dis }} \quad Q_{i}=Q_{i}^{e n}+Q_{i}^{d i s} \quad \mathcal{A}=\mathcal{A}^{e n}+\mathcal{A}^{\text {dis }}
$$

Substituting Eq. (2.9) into Eq. (2.8) and rearranging them in accordance with the energetic and the dissipative parts results in the following expression

$$
\begin{gathered}
\left(\sigma_{i j}-\rho \frac{\partial \Psi}{\partial \varepsilon_{i j}^{e}}\right) \dot{\varepsilon}_{i j}^{e}+\left(x^{e n}-\rho \frac{\partial \Psi}{\partial e^{p}}\right) \dot{e}^{p}+\left(Q_{i}^{e n}-\rho \frac{\partial \Psi}{\partial e_{, i}^{p}}\right) \dot{e}_{, i}^{p}+\left(\mathcal{A}^{e n}-\rho s-\rho \frac{\partial \Psi}{\partial \mathcal{T}}\right) \dot{\mathcal{T}} \\
+\left(\mathcal{B}_{i}-\rho \frac{\partial \Psi}{\partial \mathcal{T}_{, i}}\right) \dot{\mathcal{T}}_{, i}+x^{d i s} \dot{e}^{p}+Q_{i}^{d i s} \dot{e}_{, i}^{p}+\mathcal{A}^{d i s} \dot{\mathcal{T}}-\frac{q_{i}}{\mathcal{T}} \mathcal{T}_{, i} \geqslant 0
\end{gathered}
$$


By assuming that the fifth term in Eq. (2.10) is strictly energetic, the energetic components of the thermodynamic microforces are defined as follows

$$
\begin{aligned}
& \sigma_{i j}=\rho \frac{\partial \Psi}{\partial \varepsilon_{i j}^{e}} \quad x^{e n}=\rho \frac{\partial \Psi}{\partial e^{p}} \quad Q_{i}^{e n}=\rho \frac{\partial \Psi}{\partial e_{, i}^{p}} \\
& \mathcal{A}^{e n}=\rho\left(s+\frac{\partial \Psi}{\partial \mathcal{T}}\right) \quad \mathcal{B}_{i}=\rho \frac{\partial \Psi}{\partial \mathcal{T}_{, i}}
\end{aligned}
$$

The dissipation density per unit time $\mathcal{D}$ is then obtained as

$$
\mathcal{D}=x^{d i s} \dot{e}^{p}+Q_{i}^{d i s} \dot{e}_{, i}^{p}+\mathcal{A}^{d i s} \dot{\mathcal{T}}-\frac{q_{i}}{\mathcal{T}} \mathcal{T}_{, i} \geqslant 0
$$

The dissipative counterparts of the thermodynamic microforces are obtained from the dissipation potential $\mathcal{D}\left(\dot{e}^{p}, \dot{e}_{, i}^{p}, \dot{\mathcal{T}}, \mathcal{T}_{, i}\right)$ as follows

$$
x^{d i s}=\frac{\partial \mathcal{D}}{\partial \dot{e}^{p}} \quad Q_{i}^{d i s}=\frac{\partial \mathcal{D}}{\partial \dot{e}_{, i}^{p}} \quad \mathcal{A}^{\text {dis }}=\frac{\partial \mathcal{D}}{\partial \dot{\mathcal{T}}} \quad-\frac{q_{i}}{\mathcal{T}}=\frac{\partial \mathcal{D}}{\partial \mathcal{T}_{, i}}
$$

\subsection{Constitutive equations for the admissible potentials (grain interior)}

\subsubsection{Energetic constitutive relations}

It is important to define the proper formulation of the Helmholtz free energy $\Psi$ because it establishes the basis for the derivation of constitutive relations. In the current work, the Helmholtz free energy function is put forward as follows (Voyiadjis and Song, 2017; Voyiadjis et al., 2017)

$$
\begin{aligned}
\Psi= & \frac{1}{2 \rho} \varepsilon_{i j}^{e} E_{i j k l} \varepsilon_{k l}^{e}-\frac{\alpha^{t h}}{\rho}\left(\mathcal{T}-\mathcal{T}_{r}\right) \varepsilon_{i j}^{e} \delta_{i j}+\frac{\mathcal{H}_{0}}{\rho(r+1)}\left[1-\left(\frac{\mathcal{T}}{\mathcal{T}_{y}}\right)^{n}\right]\left(e^{p}\right)^{r+1} \\
& +\frac{\sigma_{0}}{\rho(\vartheta+1)}\left[\ell_{e n}^{2}\left(e_{, i}^{p} e_{, i}^{p}\right)\right]^{\frac{\vartheta+1}{2}}-\frac{1}{2} \frac{c_{\varepsilon}}{\mathcal{T}_{r}}\left(\mathcal{T}-\mathcal{T}_{r}\right)^{2}-\frac{1}{2 \rho} a \mathcal{T}_{, i} \mathcal{T}_{, i}
\end{aligned}
$$

where $\alpha^{\text {th }}$ is the thermal expansion coefficient, $E_{i j k l}$ is the elastic modulus tensor, $\mathcal{H}_{0}$ is the standard isotropic hardening parameter, $r(0<r<1)$ is the isotropic hardening material parameter, $\mathcal{T}_{y}$ and $n$ are the thermal material parameters, $\sigma_{0}>0$ is the stress-dimensioned scaling parameter to explain the initial slip resistance, $\ell_{e n}$ is the energetic material length scale describing the feature of short-range interaction of the GNDs, $a$ is the material constant for the isotropic heat conduction, $\vartheta$ is the parameter of governing nonlinearity of the gradient dependent defect energy, $\mathcal{T}_{r}>0$ is the reference temperature, and $c_{\varepsilon}$ is the specific heat capacity at a constant stress.

One can now obtain the energetic thermodynamic forces by using Eqs. (2.11) and (2.14) as follows

$$
\begin{aligned}
& \sigma_{i j}=E_{i j k l} \varepsilon_{k l}^{e}-\alpha^{t h}\left(\mathcal{T}-\mathcal{T}_{r}\right) \delta_{i j} \quad x^{e n}=\mathcal{H}_{0}\left[1-\left(\frac{\mathcal{T}}{\mathcal{T}_{y}}\right)^{n}\right]\left(e^{p}\right)^{r} \\
& Q_{i}^{e n}=\sigma_{0} \ell_{e n}^{2}\left[\ell_{e n}^{2}\left(e_{, k}^{p} e_{, k}^{p}\right)\right]^{\frac{\vartheta-1}{2}} e_{, i}^{p} \\
& \mathcal{A}^{e n}=\rho s-\alpha^{t h}\left(\mathcal{T}-\mathcal{T}_{r}\right) \varepsilon_{i j}^{e} \delta_{i j}-\frac{c_{\varepsilon}}{\mathcal{T}_{r}}\left(\mathcal{T}-\mathcal{T}_{r}\right)-\frac{\mathcal{H}_{0}\left(e^{p}\right)^{r+1}}{r+1} \frac{\mathcal{T}}{\mathcal{T}_{y}}\left(\frac{\mathcal{T}}{\mathcal{T}_{y}}\right)^{n-1} \\
& \mathcal{B}_{i}=-a \mathcal{T}_{, i}
\end{aligned}
$$




\subsubsection{Dissipative constitutive relations}

In this work, the following functional form of the dissipation potential is put forward

$$
\begin{aligned}
\mathcal{D}= & \sigma_{0} \sqrt{\mathcal{H}^{2}\left(e^{p}\right)+\ell_{N-G} \varepsilon^{p}}\left[1-\left(\frac{\mathcal{T}}{\mathcal{T}_{y}}\right)^{n}\right]\left(\frac{\dot{e}^{p}}{\dot{p}_{1}}\right)^{m_{1}} \dot{e}^{p} \\
& +\sigma_{0}\left[1-\left(\frac{\mathcal{T}}{\mathcal{T}_{y}}\right)^{n}\right]\left(\frac{\dot{p}}{\dot{p}_{2}}\right)^{m_{2}} \dot{p}-\frac{\varsigma}{2} \dot{\mathcal{T}}^{2}-\frac{1}{2} \frac{k(\mathcal{T})}{\mathcal{T}} \mathcal{T}_{, i} \mathcal{T}_{, i}
\end{aligned}
$$

where $\dot{p}_{1}$ and $\dot{p}_{2}$ are the non-negative reference rates, $m_{1}$ and $m_{2}$ are the non-negative rate sensitivity parameters, $\varsigma$ is the material constant characterizing the energy exchange between phonon and electron, and $k(\mathcal{T})$ is the thermal conductivity coefficient. The N-G material length scale $\ell_{N-G}$ was first introduced by Nix and Gao (1998). In the special case $\ell_{N-G}=0$ and $\mathcal{H}\left(e^{p}\right)=1$. The first term in RHS reduces to $\sigma_{0}\left(1-\left(\mathcal{T} / \mathcal{T}_{y}\right)^{n}\right)\left(\dot{e}^{p} / \dot{p}_{1}\right)^{m_{1}} \dot{e}^{p}$, a form used by Voyiadjis and Song (2017). $\varepsilon^{p}$ is a scalar measure of an effective plastic strain gradient defined by $\varepsilon^{p} \stackrel{\text { def }}{=}\left\|\alpha_{i j}\right\|=b \rho_{G}$ with the magnitude of the Burgers vector $b$, Nye dislocation density tensor $\alpha_{i j}$ and the total GNDs density $\rho_{G}$.

The parameter $\dot{p}$ is a scalar measuring the plastic strain rate gradient, which is defined by

$$
\dot{p} \stackrel{\text { def }}{=} \ell_{\text {dis }}\left\|\dot{e}_{, i}^{p}\right\|=\ell_{\text {dis }} \sqrt{\dot{e}_{, i}^{p} \dot{e}_{, i}^{p}}
$$

where $\ell_{d i s}$ is the dissipative material length scale.

The dimensionless function $\mathcal{H}\left(e^{p}\right)$ is related to the strain hardening/softening behavior. In the current work, the following form of the mixed-hardening function is adopted (Voce, 1955)

$$
\mathcal{H}\left(e^{p}\right)=1+(\chi-1)\left[1-\exp \left(-\omega e^{p}\right)\right]+\frac{\mathcal{H}_{0}}{\sigma_{0}} e^{p}
$$

where $\chi$ and $\omega$ are the material parameters. The strain hardening, strain softening and strain hardening/softening can be modeled based on the particular choices for these parameters.

Using the dissipative potential given in Eq. (2.16) along with Eq. (2.13) and considering $k(\mathcal{T}) / \mathcal{T}=k_{0}=$ const, the constitutive relations for the dissipative microforces are obtained as follows

$$
\begin{aligned}
& x^{d i s}=\sigma_{0} \sqrt{\mathcal{H}^{2}\left(e^{p}\right)+\ell_{N-G} \varepsilon^{p}}\left[1-\left(\frac{\mathcal{T}}{\mathcal{T}_{y}}\right)^{n}\right]\left(\frac{\dot{e}^{p}}{\dot{p}_{1}}\right)^{m_{1}} \\
& Q_{i}^{d i s}=\sigma_{0} \ell_{d i s}^{2}\left(m_{2}+1\right)\left[1-\left(\frac{\mathcal{T}}{\mathcal{T}_{y}}\right)^{n}\right]\left(\frac{\dot{p}}{\dot{p}_{2}}\right)^{m_{2}} \frac{\dot{e}_{, i}^{p}}{\dot{p}} \\
& \mathcal{A}^{\text {dis }}=-\varsigma \dot{\mathcal{T}} \quad \frac{q_{i}}{\mathcal{T}}=k_{0} \mathcal{T}_{, i}
\end{aligned}
$$

\subsection{Flow rule (grain interior)}

The flow rule is established based on the nonlocal microforce balance, Eq. (2.3), and strengthened by thermodynamically consistent constitutive relations for energetic and dissipative microforces. By considering the backstress in the microforce equilibrium such as $\bar{\sigma}_{i j}-\left(-Q_{k, k}^{e n}\right) N_{i j}=$ $\left(x-Q_{k, k}^{d i s}\right) N_{i j}$, one can obtain a second order partial differential flow rule as follows

$$
\begin{aligned}
\bar{\sigma}_{i j} & -\left\{-\sigma_{0} \ell_{e n}^{2}\left[\ell_{e n}^{2}\left(e_{, i}^{p} e_{, i}^{p}\right)\right]^{\frac{\vartheta-1}{2}} e_{, k k}^{p}\right\} N_{i j}=\left\{\mathcal{H}_{0}\left[1-\left(\frac{\mathcal{T}}{\mathcal{T}_{y}}\right)^{n}\right]\left(e^{p}\right)^{r}\right. \\
& +\sigma_{0} \sqrt{\mathcal{H}^{2}\left(e^{p}\right)+\ell_{N-G} \varepsilon^{p}}\left[1-\left(\frac{\mathcal{T}}{\mathcal{T}_{y}}\right)^{n}\right]\left(\frac{\dot{e}^{p}}{\dot{p}_{1}}\right)^{m_{1}} \\
- & \left.\sigma_{0} \ell_{d i s}^{2}\left(m_{2}+1\right)\left[1-\left(\frac{\mathcal{T}}{\mathcal{T}_{y}}\right)^{n}\right]\left(\frac{\dot{p}}{\dot{p}_{2}}\right)^{m_{2}} \frac{\dot{e}_{, k k}^{p}}{\dot{p}}\right\} N_{i j}
\end{aligned}
$$

where $N_{i j}$ is the direction of plastic flow given by $N_{i j}=\dot{\varepsilon}_{i j}^{p} / \dot{e}^{p}$. 


\section{Thermodynamically consistent strain gradient plasticity model for the grain boundary}

The main goal of this study is to develop a thermodynamically consistent gradient-enhanced plasticity model for the grain boundary, which should be also consistent with the one for the grain interior addressed in Section 2. Hereafter, the superscript $G B$ and the expression GB will be used to denote specific variables at the grain boundary.

\subsection{Principle of virtual power (grain boundary)}

Two grains $\mathcal{G}_{1}$ and $\mathcal{G}_{2}$ separated by the grain boundary are taken into account in this work, and the displacement field is assumed to be continuous, i.e. $u_{i}^{\mathcal{G}_{1}}=u_{i}^{\mathcal{G}_{2}}$, across the grain boundary. The internal part of the principle of virtual power for the grain boundary is assumed to depend on the GB accumulated plastic strain rates $e^{p^{G B \mathcal{G}_{1}}}$ at $S^{G B \mathcal{G}_{1}}$ and $e^{p^{G B \mathcal{G}_{2}}}$ at $S^{G B \mathcal{G}_{2}}$ in the arbitrary surface $S^{G B}$ of the grain boundary as follows

$$
\mathcal{P}^{i n t^{G B}}=\int_{S^{G B}}\left(\mathbb{M}^{G B \mathcal{G}_{1}} \dot{e}^{p^{G B \mathcal{G}_{1}}}+\mathbb{M}^{G B \mathcal{G}_{2}} \dot{e}^{p^{G B \mathcal{G}_{2}}}\right) d S^{G B}
$$

where the GB microscopic moment tractions $\mathbb{M}^{G B \mathcal{G}_{1}}$ and $\mathbb{M}^{G B \mathcal{G}_{2}}$ are assumed to expend the power over $\dot{e}^{p^{G B \mathcal{G}_{1}}}$ and $\dot{e}^{p^{G B \mathcal{G}_{2}}}$, respectively. In addition, the GB external power $\mathcal{P}^{\text {ext }}{ }^{G B}$ is expended by the macrotractions $\sigma_{i j}^{\mathcal{G}_{1}}\left(-n_{j}^{G B}\right)$ and $\sigma_{i j}^{\mathcal{G}_{2}}\left(n_{j}^{G B}\right)$ conjugate to the macroscopic velocity $\dot{u}_{i}$, and the microtractions $\mathbb{Q}_{k}^{\mathcal{G}_{1}}\left(-n_{k}^{G B}\right)$ and $\mathbb{Q}_{i}^{\mathcal{G}_{2}}\left(n_{k}^{G B}\right)$ that are conjugate to $\dot{\varepsilon}_{i j}^{G B \mathcal{G}_{1}}$ and $\dot{\varepsilon}_{i j}^{p^{G B \mathcal{G}_{2}}}$, respectively, as follows

$$
\mathcal{P}^{e x t^{G B}}=\int_{S^{G B}}\left[\left(\sigma_{i j}^{\mathcal{G}_{2}} n_{j}^{G B}-\sigma_{i j}^{\mathcal{G}_{1}} n_{j}^{G B}\right) \dot{u}_{i}+\mathbb{Q}_{k}^{\mathcal{G}_{2}} n_{k}^{G B} \dot{e}^{p^{G B \mathcal{G}_{2}}}-\mathbb{Q}_{k}^{\mathcal{G}_{1}} n_{k}^{G B} \dot{e}^{p^{G B \mathcal{G}_{1}}}\right] d S^{G B}
$$

where $\mathbf{n}^{G B}$ is the unit outward normal vector of the grain boundary surface. From $\mathcal{P}^{i n t^{G B}}=\mathcal{P}^{e x t^{G B}}$, the macro- and microforce balances for the grain boundary are obtained as follows

$$
\left(\sigma_{i j}^{\mathcal{G}_{1}}-\sigma_{i j}^{\mathcal{G}_{2}}\right) n_{j}^{G B} \quad \mathbb{M}^{G B \mathcal{G}_{1}}+\mathbb{Q}_{k}^{\mathcal{G}_{1}} n_{k}^{G B}=0 \quad \mathbb{M}^{G B \mathcal{G}_{2}}-\mathbb{Q}_{k}^{\mathcal{G}_{2}} n_{k}^{G B}=0
$$

\subsection{Laws of thermodynamics (grain boundary)}

The first and second laws of thermodynamics are considered to construct the thermodynamically consistent gradient- and temperature-enhanced framework for the grain boundary as follows

$$
\begin{aligned}
& \dot{\mathcal{E}}^{G B}=\mathbb{M}^{G B} \dot{e}^{G B}+q_{i}^{G B} n_{i}^{G B} \\
& \dot{s}^{G B} \mathcal{T}^{G B}-q_{i}^{G B} n_{i}^{G B} \geqslant 0
\end{aligned}
$$

where $\mathcal{E}^{G B}$ is the GB surface energy density, $q_{i}^{G B}$ is the GB heat flux vector and $s^{G B}$ is the surface density of entropy of the grain boundary.

\subsection{Energetic and dissipative thermodynamic microforces (grain boundary)}

By using the time derivative of the equation, $\Psi^{G B}=\mathcal{E}^{G B}-\mathcal{T}^{G B}{ }_{s}{ }^{G B}$, and substituting it into Eqs. (3.4), the following Clausius-Duhem inequality for the grain boundary is obtained

$$
\mathbb{M}^{G B} \dot{e}^{p^{G B}}-\dot{\Psi}^{G B}-s^{G B} \dot{\mathcal{T}}^{G B} \geqslant 0
$$


Suppose the isothermal condition for the grain boundary $\left(\dot{\mathcal{T}}^{G B}=0\right)$ and the Helmholtz free energy for the grain boundary is given by $\Psi^{G B}=\Psi^{G B}\left(e^{p^{G B}}\right)$. Substituting the time derivative of $\Psi^{G B}$ into Eq. (3.5) gives the following inequality

$$
\mathbb{M}^{G B} \dot{e}^{p^{G B}}-\rho \frac{\partial \Psi^{G B}}{\partial e^{p^{G B}}} \dot{e}^{p^{G B}} \geqslant 0
$$

The GB thermodynamic microforce quantitity $\mathbb{M}^{G B}$ is further assumed to be decomposed into the energy and dissipative components such as $\mathbb{M}^{G B}=\mathbb{M}^{G B, e n}+\mathbb{M}^{G B, d i s}$. The components $\mathbb{M}^{G B, e n}$ and $\mathbb{M}^{G B, d i s}$ indicate the mechanisms for the pre- and post-slip transfer, and thus involve the plastic strain at the grain boundary prior to the slip transfer $e^{p^{G B(p r e)}}$ and the one after the slip transfer $e^{p^{G B(p o s t)}}$, respectively, $\left(e^{p^{G B}}=e^{p^{G B(p r e)}}+e^{p^{G B(p o s t)}}\right)$. From Eq. (3.6)

$$
\left(\mathbb{M}^{G B, e n}-\rho \frac{\partial \Psi^{G B}}{\partial e^{p^{G B}}}\right) \dot{e}^{p^{G B}}+\mathbb{M}^{G B, d i s} \dot{e}^{p^{G B}} \geqslant 0
$$

The GB energetic microforce can be obtained as

$$
\mathbb{M}^{G B, e n}=\rho \frac{\partial \Psi^{G B}}{\partial e^{p^{G B}}}
$$

Hence the GB dissipative microforce can then be obtained as

$$
\mathbb{M}^{G B, d i s}=\frac{\partial \mathcal{D}^{G B}}{\partial \dot{e}^{p^{G B}}}
$$

where $\mathcal{D}^{G B}$ is the non-negative dissipation density per unit time for the grain boundary, given by $\mathcal{D}^{G B}=\mathbb{M}^{G B, d i s} \dot{e}^{p^{G B}} \geqslant 0$. This non-negative plastic dissipation condition can be satisfied when the GB plastic dissipation potential is a convex function of the GB accumulated plastic strain rate.

\subsection{Energetic and dissipative thermodynamic microforces (grain boundary)}

In this work, it is assumed, following Fredriksson and Gudmundson (2007), that the GB Helmholtz free energy per unit surface has the form of a general power law as follows

$$
\Psi^{G B}\left(e^{p^{G B}}\right)=\frac{1}{2} G \ell_{e n}^{G B}\left(e^{p^{G B(p r e)}}\right)^{2}
$$

where $G$ is the shear modulus in the case of isotropic linear elasticity, $\ell_{e n}^{G B}$ is the GB energetic length scale. By substituting Eq. (3.10) into Eq. (3.8), the GB energetic microforce quantity can be obtained as follows

$$
\mathbb{M}^{G B, e n}=G \ell_{e n}^{G B} e^{p^{G B(p r e)}}
$$

Note that $\mathbb{M}^{G B, e n}$ is independent of the plastic strain rate and temperature since this variable comes from the recoverable stored energy.

Meanwhile, two major factors might be identified affecting energy dissipation when the dislocations move in the grain boundary area (Aifantis and Willis, 2005). When the dislocations encounter a grain boundary, they pile up there. Slip can transmit to the adjacent grain only when the stress field ahead of the pileup is high enough. Direct observation of the process using transmission electron microscopy (TEM) also shows that the main mechanisms for the aforementioned slip transmission are dislocation absorption and re-emission for low angle boundaries (Soer et al., 2005) and the dislocation nucleation in the adjacent grain for high angle boundaries (Ohmura et al., 2004), respectively. As soon as deformation initiates in the adjacent grain, the 
grain boundary begins to deform and the plastic strain on the grain boundary increases. The energy associated with the deformation of the grain boundary in this case is taken to be mainly due to energy dissipation as the dislocations move in the grain boundary region. In addition, considering the resistance force to dislocation motion being temperature and rate dependent, this energy dissipation can be taken as a linear function of the GB plastic strain.

Moreover, a change in the grain boundary area can also affect the energy dissipation. The macroscopic accumulated plastic strain at the grain boundary, $e^{p^{G B}}$, can be related to microscopic deformation of the grain boundary through the root-mean-square of the gradient of this deformation. In addition, the energy change after the grain boundary has yielded, i.e. the onset of slip transmission, can be approximated by a quadratic function of the aforementioned displacement gradient at microscale and hence the GB plastic strain at macroscale.

Combining both the aforementioned mechanisms, i.e. a change in the grain boundary area and deformation of the grain boundary due to the dislocation movement, involved in the energy dissipation due to plastic strain transfer across the grain boundary, one can postulate the following generalized expression for the GB dissipation potential

$$
\mathcal{D}^{G B}=\frac{\ell_{\text {dis }}^{G B}}{m^{G B}+1}\left(\sigma_{0}^{G B}+\mathcal{H}_{0}^{G B} e^{p^{G B(p o s t)}}\right)\left(1-\frac{\mathcal{T}^{G B}}{\mathcal{T}_{y}^{G B}}\right)^{n^{G B}}\left(\frac{\dot{e}^{p^{G B(p o s t)}}}{\dot{p}^{G B}}\right)^{m^{G B}} \dot{e}^{p^{G B(p o s t)}} \geqslant 0
$$

where $\ell_{\text {dis }}^{G B}$ is the GB dissipative length scale, $m^{G B}$ and $\dot{p}^{G B}$ are the viscous related material parameters, $\sigma_{0}^{G B}$ is a constant accounting for the GB yield stress, $\mathcal{H}_{0}^{G B}$ is the GB hardening parameter, $\mathcal{T}_{y}^{G B}$ is the scale-independent GB thermal parameter at the onset of yield, $n^{G B}$ is the GB thermal parameter. The temperature- and rate-dependency of the GB energy are shown respectively in terms $\left(1-\mathcal{T}^{G B} / \mathcal{T}_{y}^{G B}\right)^{n^{G B}}$ and $\left(\dot{e}^{p^{G B(p o s t)}} / \dot{p}^{G B}\right)^{m^{G B}}$.

By using Eqs. (3.9) and (3.12), the GB dissipative microforce $\mathbb{M}^{G B \text {,dis }}$ can be obtained as

$$
\mathbb{M}^{G B, d i s}=\ell_{\text {dis }}^{G B}\left(\sigma_{0}^{G B}+\mathcal{H}_{0}^{G B} e^{p^{G B(p o s t)}}\right)\left(1-\frac{\mathcal{T}^{G B}}{\mathcal{T}_{y}^{G B}}\right)^{n^{G B}}\left(\frac{\dot{e}^{p^{G B(p o s t)}}}{\dot{p}^{G B}}\right)^{m^{G B}}
$$

Therefore, the GB thermodynamic microforce $\mathbb{M}^{G B}$ can be obtained as

$$
\begin{aligned}
\mathbb{M}^{G B} & =G \ell_{e n}^{G B} e^{p^{G B(p r e)}} \\
& +\ell_{\text {dis }}^{G B}\left(\sigma_{0}^{G B}+\mathcal{H}_{0}^{G B} e^{p^{G B(p o s t)}}\right)\left(1-\frac{\mathcal{T}^{G B}}{\mathcal{T}_{y}^{G B}}\right)^{n^{G B}}\left(\frac{\dot{e}^{p^{G B(p o s t)}}}{\dot{p}^{G B}}\right)^{m^{G B}}
\end{aligned}
$$

It can be seen from Eq. (3.14) that the grain boundary may act like a free surface, i.e. microscopically free boundary condition, when $\ell_{e n}^{G B}=\ell_{d i s}^{G B}=0$. On the other hand, the microscopically hard boundary condition can be compelled under the conditions $\ell_{e n}^{G B} \rightarrow \infty$ and $\ell_{d i s}^{G B} \rightarrow \infty$.

\subsection{Flow rule (grain boundary)}

The flow rule for the grain boundary can be derived by substituting Eq. (3.14) into the microforce balances for the grain boundary, Eq. (3.3), such as:

- for $S^{G B \mathcal{G}_{1}}$

$$
\begin{gathered}
\left\{\sigma_{0} \ell_{e n}^{2}\left[\ell_{e n}^{2}\left(e_{, k}^{p} e_{, k}^{p}\right)\right]^{\frac{\vartheta-1}{2}} e_{, i}^{p}+\sigma_{0} \ell_{d i s}^{2}\left(m_{2}+1\right)\left[1-\left(\frac{\mathcal{T}}{\mathcal{T}_{y}}\right)^{n}\right]\left(\frac{\dot{p}}{\dot{p}_{2}}\right)^{m_{2}} \frac{\dot{e}_{, i}^{p}}{\dot{p}}\right\} n_{k}^{G B}+G \ell_{\text {en }}^{G B} e^{p^{G B(p r e) ~}} \\
=-\ell_{d i s}^{G B}\left(\sigma_{0}^{G B}+\mathcal{H}_{0}^{G B} e^{p^{G B(p o s t)}}\right)\left(1-\frac{\mathcal{T}^{G B}}{\mathcal{T}_{y}^{G B}}\right)^{n^{G B}}\left(\frac{\dot{e}^{p^{G B(p o s t)}}}{\dot{p}^{G B}}\right)^{m^{G B}}
\end{gathered}
$$


- for $S^{G B \mathcal{G}_{2}}$

$$
\begin{gathered}
\left\{\sigma_{0} \ell_{\text {en }}^{2}\left[\ell_{e n}^{2}\left(e_{, k}^{p} e_{, k}^{p}\right)\right]^{\frac{\vartheta-1}{2}} e_{, i}^{p}+\sigma_{0} \ell_{d i s}^{2}\left(m_{2}+1\right)\left[1-\left(\frac{\mathcal{T}}{\mathcal{T}_{y}}\right)^{n}\right]\left(\frac{\dot{p}}{\dot{p}_{2}}\right)^{m_{2}} \frac{\dot{e}_{, i}^{p}}{\dot{p}}\right\} n_{k}^{G B}-G \ell_{e n}^{G B} e^{p^{G B(p r e)}} \\
=\ell_{\text {dis }}^{G B}\left(\sigma_{0}^{G B}+\mathcal{H}_{0}^{G B} e^{p^{G B(p o s t)}}\right)\left(1-\frac{\mathcal{T}^{G B}}{\mathcal{T}_{y}^{G B}}\right)^{n^{G B}}\left(\frac{\dot{e}^{p^{G B(p o s t)}}}{\dot{p}^{G B}}\right)^{m^{G B}}
\end{gathered}
$$

where the second term in LHS of both equations represents the backstress. Note that, in a general case, the grain boundary model parameters are not identical on each side, however in this work, the same values are assumed to be considered for simplification.

Considering the GB flow rules as the boundary conditions of the grain interior flow rule, Eq. (2.20), results in a yield condition accounting for the temperature and rate dependent barrier effect of grain boundaries on the plastic slip and, consequently, the influence on the GNDs evolution in the grain interior.

\section{Finite element implementation of the proposed SGP model}

A two-dimensional finite element model for the derived grain interior/boundary flow rules is developed to account for the size dependent response for microscopic structures. In this finite element solution, the plastic strain field $e^{p}$ as well as the displacement field $u_{i}$ are discretized independently and both the fields are taken as fundamental unknown nodal degrees of freedom. The increments in the nodal displacement and plastic strains can be obtained by computing the system of linear equations shown in Eq. (4.1)

$$
\underbrace{\left[\begin{array}{ll}
K_{u_{i} u_{k}}^{\Omega_{e l}} & K_{u_{i} e^{p}}^{\Omega_{e l}} \\
K_{e^{p} u_{k}}^{\Omega_{e l}} & K_{e^{p} e^{p}}^{\Omega_{e}}
\end{array}\right]}_{\mathbf{K}^{\Omega_{e l}}}\left\{\begin{array}{c}
\left(\Delta \mathcal{U}_{u_{k}}\right)_{\eta} \\
\left(\Delta \mathcal{E}_{e}\right)_{\eta}
\end{array}\right\}=\left\{\begin{array}{l}
\left(\mathbb{R}_{u_{i}}\right)_{\eta} \\
\left(\mathbb{R}_{e}^{p}\right)_{\eta}
\end{array}\right\}
$$

where $\mathbf{K}^{\Omega_{e l}}$ is the Jacobian (stiffness) matrix. The nodal displacement and the plastic strain in the iteration $\zeta$ at the node $\eta$ are expressed as $\left(\mathcal{U}_{u_{i}}^{\zeta}\right)_{\eta}$ and $\left(\mathcal{E}_{e^{p}}^{\zeta}\right)_{\eta}$, respectively, and their increments are calculated by $\left(\Delta \mathcal{U}_{u_{k}}\right)_{\eta}=\left(\mathcal{U}_{u_{i}}^{\zeta+1}\right)_{\eta}-\left(\mathcal{U}_{u_{i}}^{\zeta}\right)_{\eta},\left(\Delta \mathcal{E}_{e^{p}}\right)_{\eta}=\left(\mathcal{E}_{e^{p}}^{\zeta+1}\right)_{\eta}-\left(\mathcal{E}_{e^{p}}^{\zeta}\right)_{\eta} \cdot\left(\mathbb{R}_{u_{i}}\right)_{\eta}$ and $\left(\mathbb{R}_{e^{p}}\right)_{\eta}$ are the nodal residuals for the displacement and plastic strain at the node $\eta$, respectively. The system of linear equations is solved via the user-defined element subroutine (UEL) in ABAQUS/standard using the Newton-Raphson iterative method.

Each component of the Jacobian matrix can be obtained respectively as follows

$$
\begin{aligned}
& K_{u_{i} u_{k}}^{\Omega_{e l}}=-\left.\frac{\partial \mathbb{R}_{u_{i}}}{\partial \mathcal{U}_{u_{k}}^{\eta}}\right|_{\mathcal{U}_{u_{i}}^{\zeta}}=\int_{\Omega_{e l}}\left(E_{i j k l} \frac{\partial \mathbb{N}_{u}^{\eta}}{\partial x_{j}} \frac{\partial \mathbb{N}_{u}^{\eta}}{\partial x_{l}}\right) d V \\
& K_{u_{i} e^{p}}^{\Omega_{e l}}=-\left.\frac{\partial \mathbb{R}_{u_{i}}}{\partial \mathcal{E}_{e^{p}}^{\eta}}\right|_{\mathcal{E}_{e^{p}}^{\zeta}}=\int_{\Omega_{e l}}\left(E_{i j k l} \frac{e^{p}}{\varepsilon_{k l}} \frac{\partial \mathbb{N}_{u}^{\eta}}{\partial x_{j}} \mathbb{N}_{e^{p}}^{\eta}\right) d V \\
& K_{e^{p} u_{k}}^{\Omega_{e l}}=-\left.\frac{\partial \mathbb{R}_{e^{p}}}{\partial \mathcal{U}_{u_{k}}^{\eta}}\right|_{\mathcal{U}_{u_{i}}^{\zeta}}=\int_{\Omega_{e l}}\left(E_{i j k l} \frac{e^{p}}{\varepsilon_{i j}} \mathbb{N}_{u}^{\eta} \frac{\partial \mathbb{N}_{e^{p}}^{\eta}}{\partial x_{l}}\right) d V
\end{aligned}
$$

and 


$$
\begin{aligned}
K_{e^{p} e^{p}}^{\Omega_{e l}} & =-\left.\frac{\partial \mathbb{R}_{e^{p}}}{\partial \mathcal{E}_{e^{p}}^{\eta}}\right|_{\mathcal{E}_{e^{p}}^{\zeta}}=\int_{\Omega_{e l}}\left\{\left(r \mathcal{H}_{0}\left(e^{p}\right)^{r}+\mathcal{H}_{0}\left(\frac{\dot{e}^{p}}{\dot{p}_{1}}\right)^{m_{1}}\right.\right. \\
& \left.+\sigma_{0} \sqrt{\mathcal{H}^{2}\left(e^{p}\right)+\ell_{N-G} \varepsilon^{p}} \frac{m_{1} \dot{e}^{p^{m_{1}-1}}}{\dot{p}_{1}^{m_{1}} \Delta t}\right)\left[1-\left(\frac{\mathcal{T}}{\mathcal{T}_{y}}\right)^{n}\right] \mathbb{N}_{e^{p}}^{\eta} \mathbb{N}_{e^{p}}^{\eta} \\
& +\sigma_{0} \ell_{e n}^{2}\left[\ell_{e n}^{2}\left(e_{, k}^{p} e_{, k}^{p}\right)\right]^{\frac{\vartheta-1}{2}} \frac{\partial \mathbb{N}_{e^{p}}^{\eta}}{\partial x_{j}} \frac{\partial \mathbb{N}_{e^{p}}^{\eta}}{\partial x_{j}} \\
& +\sigma_{0} \ell_{d i s}^{4}\left(m_{2}^{2}-1\right)\left[1-\left(\frac{\mathcal{T}}{\mathcal{T}_{y}}\right)^{n}\right] \frac{\dot{p}^{m_{2}-3}}{\dot{p}_{2}^{m_{2}} \Delta t}\left(\dot{e}_{, j}^{p} \frac{\partial \mathbb{N}_{e^{p}}^{\eta}}{\partial x_{j}}\right)\left(\dot{e}_{, j}^{p} \frac{\partial \mathbb{N}_{e^{p}}^{\eta}}{\partial x_{j}}\right) \\
& \left.+\sigma_{0} \ell_{d i s}^{2}\left(m_{2}+1\right)\left[1-\left(\frac{\mathcal{T}}{\mathcal{T}_{y}}\right)^{n}\right] \frac{\dot{p}^{m_{2}-1}}{\dot{p}_{2}^{m_{2}} \Delta t} \frac{\partial \mathbb{N}_{e^{p}}^{\eta}}{\partial x_{j}} \frac{\partial \mathbb{N}_{e^{p}}^{\eta}}{\partial x_{j}}\right\} d V \\
& -\left[G \ell_{e n}^{G B}+\ell_{d i s}^{G B} \frac{\sigma_{0}^{G B}+\mathcal{H}_{0}^{G B} e^{p^{G B(p o s t)}}}{\left(\Delta t \dot{p}^{G B}\right)^{m^{G B}}}\left(1-\frac{\mathcal{T}^{G B}}{\mathcal{T}_{y}^{G B}}\right)^{n^{G B}} \dot{e}^{p^{G B(p o s t)} m^{G B}-1}\right] \mathbb{N}_{e^{p}}^{\eta} \mathbb{N}_{e^{p}}^{\eta}
\end{aligned}
$$

where $\Delta t$ is the time step. The grain boundary terms in Eq. (4.3) are only applied for nodes on the grain boundary area.

\section{Numerical examples}

The assumption of the microscopically hard boundary condition is used in the authors' previous work (Song and Voyiadjis, 2018). In this work, the governing differential equation is solved by imposing the proposed grain boundary flow rule to account for the deformable grain boundary. Furthermore, the characteristics of the proposed strain gradient plasticity theory incorporating the flow rules of both the grain interior and the grain boundary is addressed in this Section by solving the shear problem of a square plate with an edge of $L$. A schematic illustration of the problem, initial conditions and macroscopic and microscopic boundary conditions as well as the grain boundary area are shown in Fig. 1. The parameter $u^{+}(t)$ represents the prescribed displacement. The whole square is meshed using $1600(40 \times 40)$ elements and split into $16(4 \times 4)$ grains by the grain boundary area, which is indicated by bold lines.

(a)

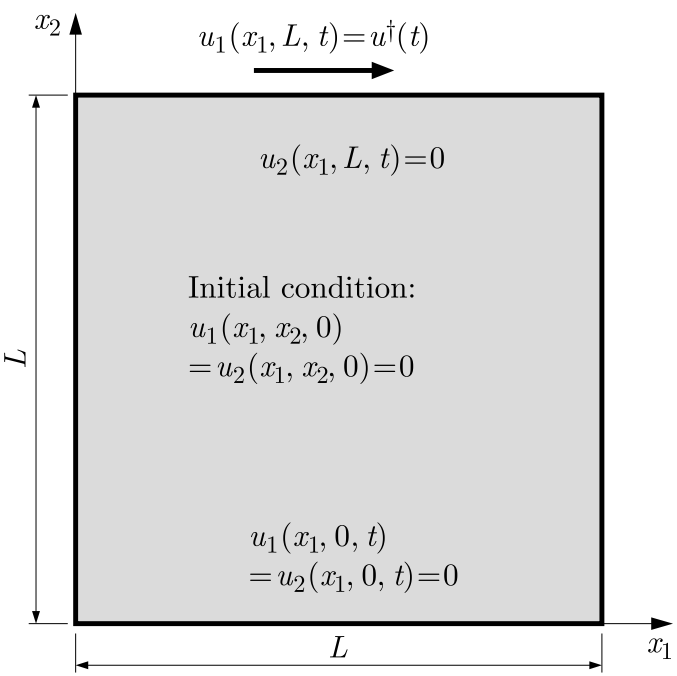

(b)

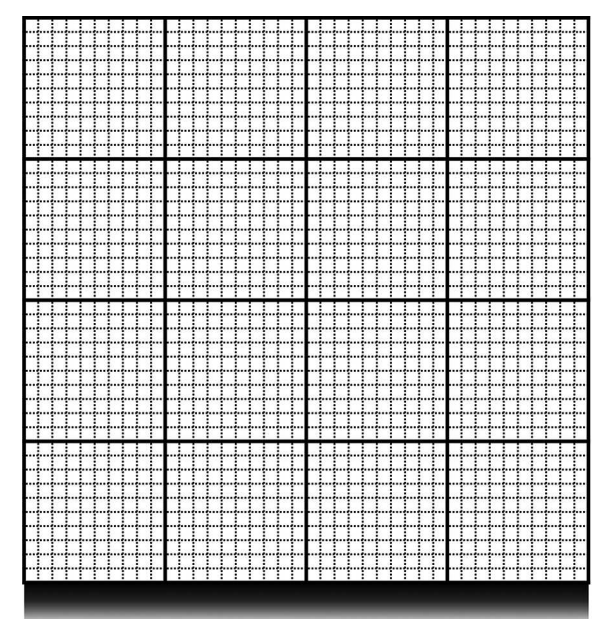

Fig. 1. The schematic illustration of the simple shear problem: (a) macroscopic, microscopic boundary conditions, and initial conditions, (b) $4 \times 4$ grains 
The following material parameters are used in this Section unless stated otherwise: $E=110 \mathrm{GPa}, \nu=0.343, \rho=8.960 \mathrm{~g} \cdot \mathrm{cm}^{-3}, c_{\varepsilon}=0.385 \mathrm{~J} /(\mathrm{g} \cdot \mathrm{K}), \quad \alpha^{t h}=16.0 \mu \mathrm{m} /(\mathrm{m} \cdot \mathrm{K})$, $\dot{p}_{1}=\dot{p}_{2}=0.04 \mathrm{~s}^{-1}, r=0.6, m_{1}=0.05, m_{2}=0.2, \mathcal{T}_{y}=1358 \mathrm{~K}, n=0.3, \sigma_{0}=195 \mathrm{MPa}$, $\mathcal{H}_{0}=0 \mathrm{MPa}, \sigma_{0}^{G B}=300 \mathrm{MPa}, \mathcal{H}_{0}^{G B}=300 \mathrm{MPa}, \dot{p}^{G B}=0.04 \mathrm{~s}^{-1}, m^{G B}=1, \mathcal{T}_{y}^{G B}=700 \mathrm{~K}$, $n^{G B}=0.4$.

As mentioned in Section 3.4, the microscopically free and hard boundary conditions at the grain boundary can be introduced respectively by setting $\ell_{e n}^{G B}=\ell_{d i s}^{G B}=0$ and $\ell_{e n}^{G B} \rightarrow \infty$, $\ell_{d i s}^{G B} \rightarrow \infty$. Firstly, the validity of these conditions is examined in this work. Next, a direct comparison between the classical plasticity theory $\left(\ell_{e n} / L=\ell_{d i s} / L=\ell_{N-G} / L=0.0\right)$ and the gradient-enhanced plasticity theory $\left(\ell_{e n} / L=\ell_{\text {dis }} / L=\ell_{N-G} / L=0.1\right)$ is given in order to check the ability of the proposed flow rule on the size effect. The numerical results in terms of the accumulated plastic strain profile and the stress-strain curves are shown in Figs. 2 and 3. The

(a)

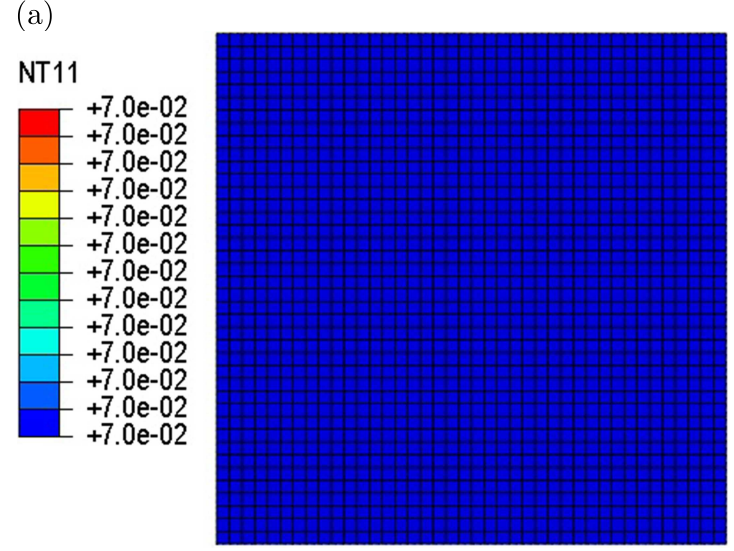

(c)

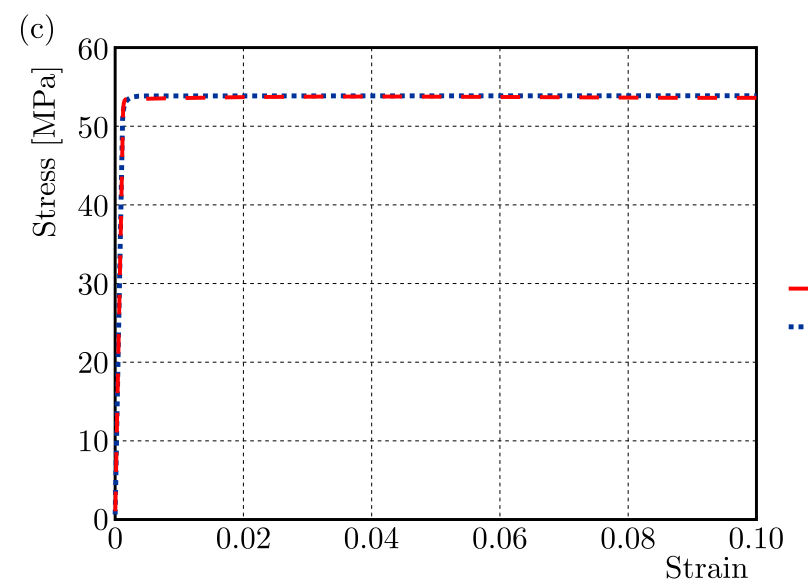

Fig. 2. Classical plasticity theory $\left(\ell_{e n} / L=\ell_{\text {dis }} / L=\ell_{N-G} / L=0\right)$. Distributions of the accumulated plastic strain with: (a) microscopically free $\left(\ell_{e n}^{G B}=\ell_{d i s}^{G B}=0\right)$, and (b) microscopically hard boundary conditions $\left(\ell_{e n}^{G B} \rightarrow \infty, \ell_{d i s}^{G B} \rightarrow \infty\right)$, and (c) stress-strain responses

terminology "NT11" in Figs. 2 and 3 indicates the accumulated plastic strain. As can be seen in these figures, the microscopically free and hard boundary conditions are well captured under the classical plasticity theory as well as the gradient-enhanced plasticity theory. In addition, in Fig. 2c, no size effect is observed in the classical plasticity theory with varying normalized material length scales as expected. In Fig. 3c, on the other hand, strain hardening and strengthening are more pronounced as the dimensions of the shear plate height are reduced $\left(\ell_{e n}^{G B} / L \rightarrow \infty\right.$, $\left.\ell_{\text {dis }}^{G B} / L \rightarrow \infty\right)$ 
(a)

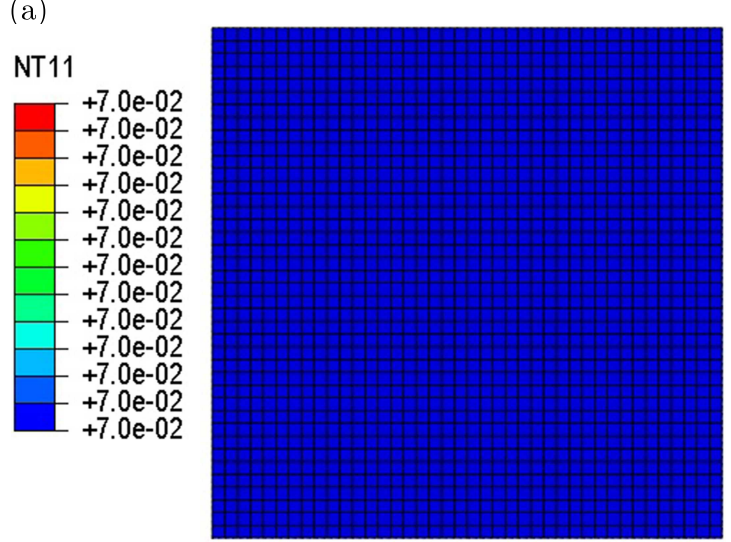

(b)

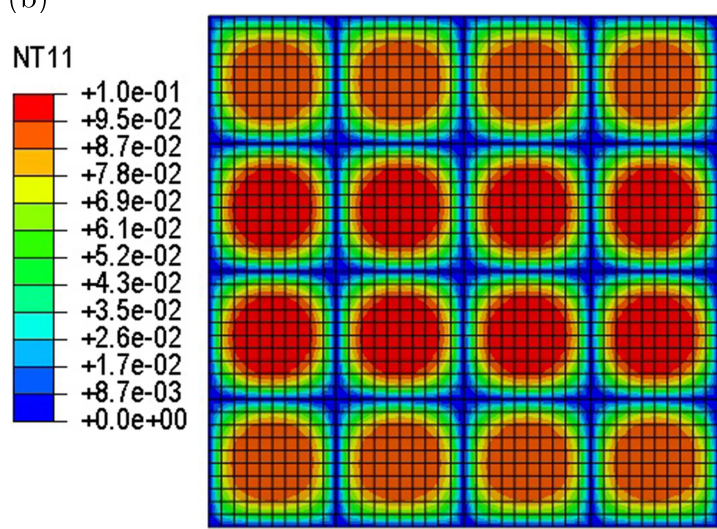

(c)

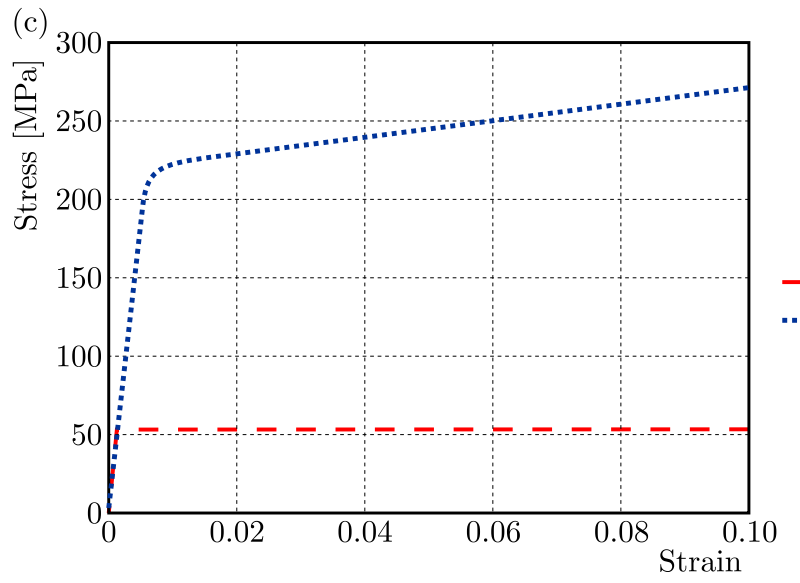

- - Microfree boundary condition

......... Microhard boundary condition

Fig. 3. Strain gradient plasticity theory $\left(\ell_{e n} / L=\ell_{d i s} / L=\ell_{N-G} / L=0.1\right)$. Distributions of the accumulated plastic strain with: (a) microscopically free $\left(\ell_{e n}^{G B}=\ell_{d i s}^{G B}=0\right)$, and (b) microscopically hard boundary conditions $\left(\ell_{e n}^{G B} \rightarrow \infty, \ell_{d i s}^{G B} \rightarrow \infty\right)$, and (c) stress-strain responses

In Fig. 4 , the effects of each material length scale parameter, i.e. $\ell_{e n}, \ell_{d i s}$ and $\ell_{N-G}$, along with the microscopically hard boundary condition are also examined through the profile of the accumulated plastic strain. In addition, the contributions of each length scale parameter on the stress-strain responses are shown in Fig. 4c.

Variations in the stress-strain responses and evolutions of the maximum temperature are investigated for various values of the normalized energetic and dissipative grain boundary material length scales as shown in Figs. 5 and 6 . It is assumed by setting $\ell_{d i s}^{G B} / \ell_{d i s}=0$ that all plastic work at the grain boundary is stored as surface energy which depends on the plastic strain state at the surface. In this case, $\ell_{e n}^{G B} / \ell_{e n}$ reflects the grain boundary resistance to plastic deformation. Figures $5 \mathrm{~b}$ and $6 \mathrm{~b}$ show the size effects on the strain hardening and temperature evolution due to the grain boundary energetic length scale, and it is more pronounced in the more strongly constrained material, i.e. increasing $\ell_{e n}^{G B} / \ell_{e n}$. On the other hand, by setting $\ell_{e n}^{G B} / \ell_{e n}=0$, it is assumed that the work performed at the grain boundary is dissipated in the absence of surface energy. In this case, $\ell_{d i s}^{G B} / \ell_{d i s}$ reflects the grain boundary resistance to slip transfer. As can be seen in Fig. 5c, the initial yield strength increases without strain hardening as $\ell_{d i s}^{G B} / \ell_{\text {dis }}$ increases.

\section{Conclusions}

The two-dimensional finite element model for the thermodynamically consistent thermomechanical coupled gradient enhanced plasticity theory is proposed on the basis of the concept 
(a)

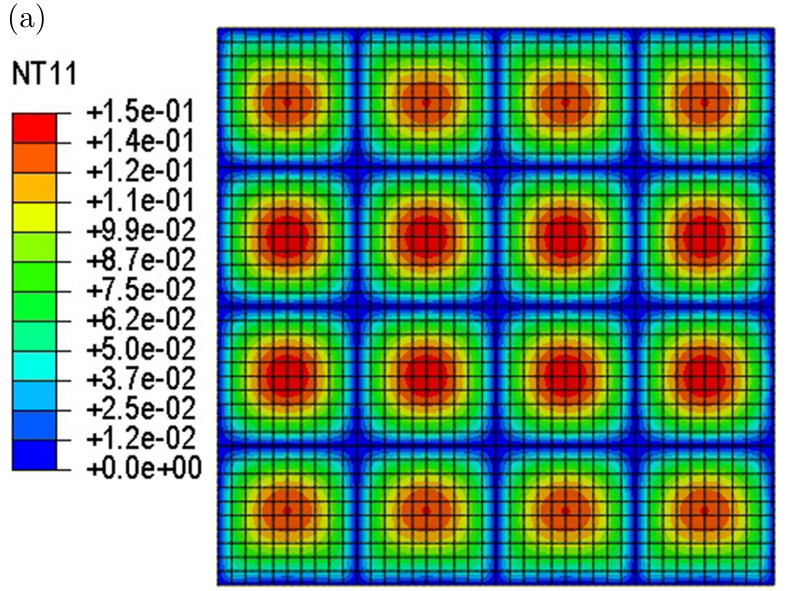

(c)

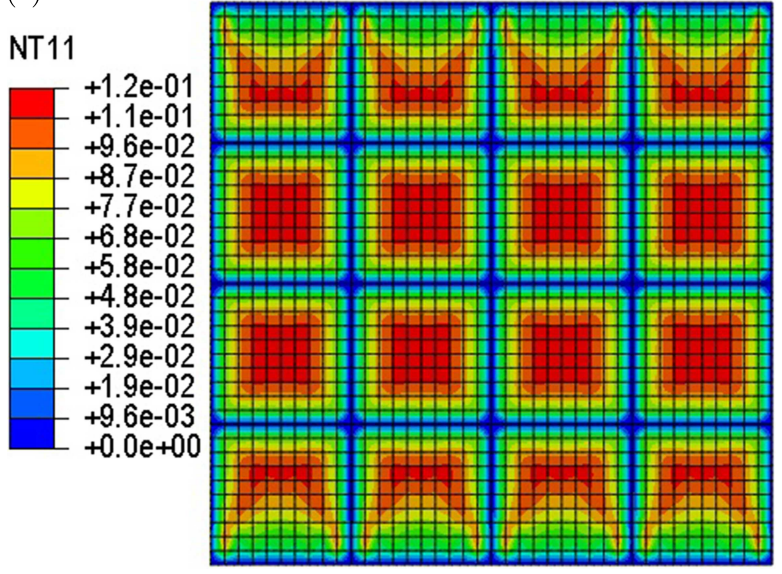

(b)

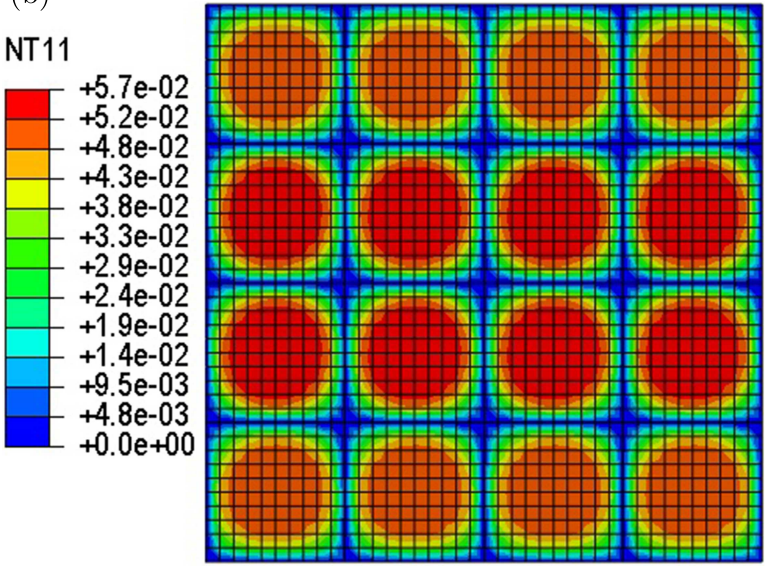

(d)

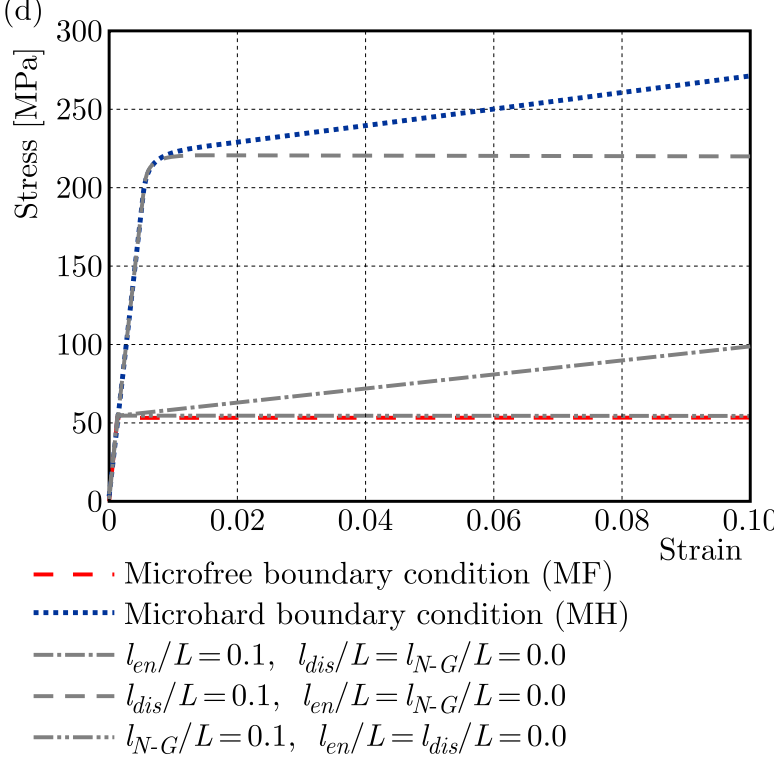

Fig. 4. Distributions of the accumulated plastic strain with the microscopically hard boundary condition $\left(\ell_{\text {en }}^{G B} / L \rightarrow \infty, \ell_{\text {dis }}^{G B} / L \rightarrow \infty\right)$ under: (a) energetic length scale only $\left(\ell_{\text {en }} / L=0.1, \ell_{\text {dis }} / L=\ell_{N-G} / L=0\right)$,

(b) dissipative length scale only $\left(\ell_{d i s} / L=0.1, \ell_{e n} / L=\ell_{N-G} / L=0\right)$, (c) N-G length scale only $\left(\ell_{N-G} / L=0.1, \ell_{e n} / L=\ell_{\text {dis }} / L=0\right)$, and (d) stress-strain responses

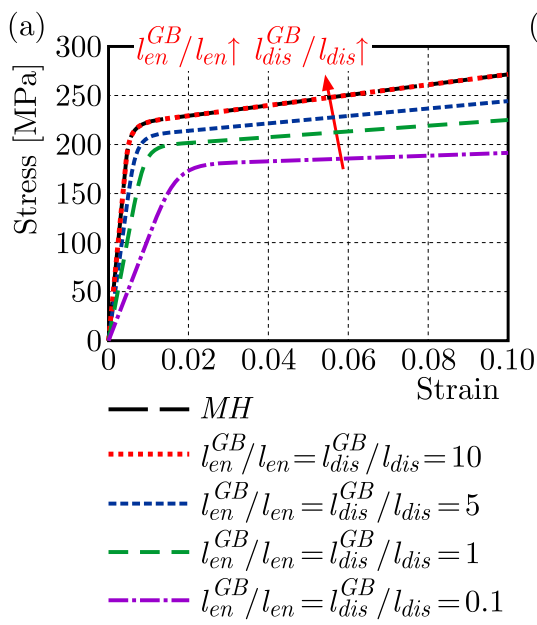

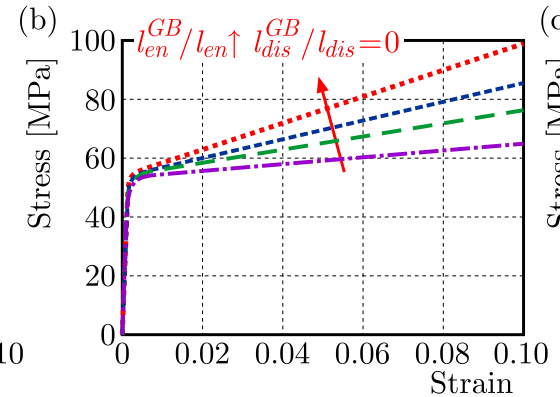

$$
\begin{aligned}
& \ldots \ldots \ldots l_{e n}^{G B} / l_{e n}=10 \quad l_{\text {dis }}^{G B} / l_{d i s}=0 \\
& l_{\text {en }}^{G B} / l_{\text {en }}=5 \quad l_{\text {dis }}^{G B} / l_{\text {dis }}=0 \\
& ---l_{\text {en }}^{G B} / l_{\text {en }}=1 \quad l_{\text {dis }}^{G B} / l_{\text {dis }}=0 \\
& \text {-.-. } l_{\text {en }}^{G B} / l_{\text {en }}=0.1 l_{\text {dis }}^{G B} / l_{\text {dis }}=0
\end{aligned}
$$

Fig. 5. Distributions of the accumulated plastic strain according to various values of $\ell_{e n}^{G B} / \ell_{e n}$ and $\ell_{d i s}^{G B} / \ell_{d i s}$ : (a) combined $\ell_{e n}^{G B}$ and $\ell_{d i s}^{G B}$, (b) $\ell_{e n}^{G B}$ only, and (c) $\ell_{d i s}^{G B}$ only 
(a)

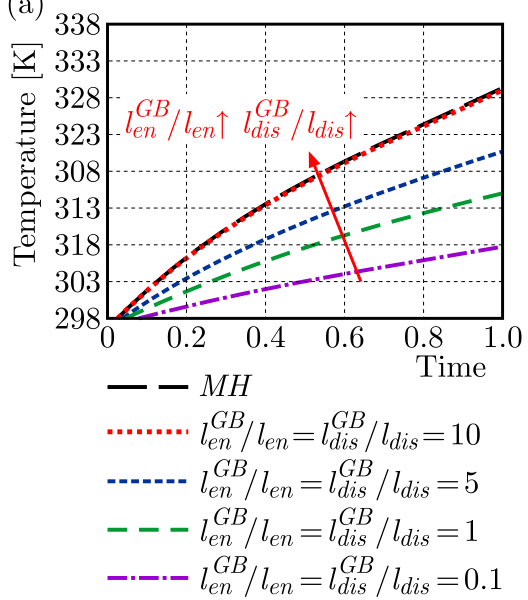

(b)

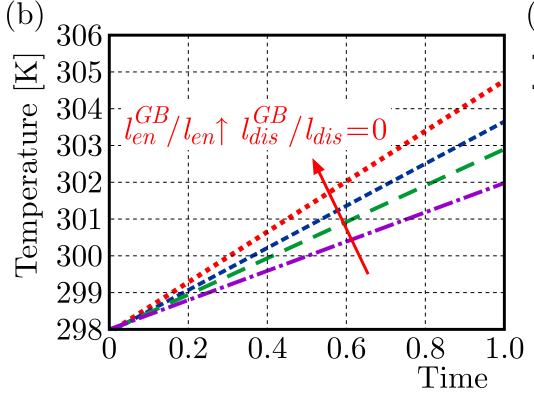

$$
\begin{aligned}
& \ldots \ldots \ldots l_{e n}^{G B} / l_{e n}=10 \quad l_{\text {dis }}^{G B} / l_{\text {dis }}=0 \\
& l_{\text {en }}^{G B} / l_{\text {en }}=5 \quad l_{\text {dis }}^{G B} / l_{\text {dis }}=0 \\
& ---l_{e n}^{G B} / l_{e n}=1 \quad l_{d i s}^{G B} / l_{d i s}=0 \\
& -\cdot-\cdot l_{\text {en }}^{G B} / l_{\text {en }}=0.1 l_{\text {dis }}^{G B} / l_{\text {dis }}=0
\end{aligned}
$$

Fig. 6. Evolutions of the maximum temperature according to various values of $\ell_{e n}^{G B} / \ell_{e n}$ and $\ell_{d i s}^{G B} / \ell_{d i s}$ : (a) combined $\ell_{e n}^{G B}$ and $\ell_{d i s}^{G B}$, (b) $\ell_{e n}^{G B}$ only, and (c) $\ell_{d i s}^{G B}$ only

of dislocation interaction mechanisms and thermal activation energy. The thermodynamic microstresses for the grain interior and grain boundary are respectively assumed to be divided into two components, i.e. energetic and dissipative components which, in turn, both energetic and dissipative material length scale parameters are incorporated in the governing constitutive equations and flow rules for both areas. These thermodynamic microstresses can be respectively obtained in a direct way from the Helmholtz free energy and the rate of dissipation potential by taking the maximum entropy production into account. In particular, the concept of GNDs density is additionally employed in the grain interior to interpret the microstructural strengthening mechanisms induced by the nonhomogeneous deformation. Correspondingly, the model in this work incorporates the terms related to GNDs-induced strengthening and the additional material length scale parameter.

In order to investigate the characteristics of the proposed strain gradient plasticity theory incorporating the flow rules for both the grain interior and grain boundary, the shear problem of a square plate is solved in this work. The microscopically free and hard boundary conditions are well captured under the classical plasticity theory as well as the gradient-enhanced plasticity theory by using the proposed grain boundary flow rule. In addition, the size effects on the stress-strain responses and the evolutions of the maximum temperature are well observed with the cases of (a) combined $\ell_{e n}^{G B}$ and $\ell_{d i s}^{G B}$, (b) $\ell_{e n}^{G B}$ only, and (c) $\ell_{d i s}^{G B}$ only.

\section{Acknowledgements}

The financial support provided by a grant from the National Science Foundation EPSCoR CIMM (grant number \#OIA-1541079) is gratefully acknowledged.

\section{References}

1. ABAQUS, 2012, User's Manual (Version 6.12). Dassault Systemes Simulia Corp., Providence, RI, USA

2. Aifantis K.E., Willis J.R., 2005, The role of interfaces in enhancing the yield strength of composites and polycrystals, Journal of the Mechanics and Physics of Solids, 53, 5, 1047-1070

3. Cermelli P., Gurtin M.E., 2002, Geometrically necessary dislocations in viscoplastic single crystals and bicrystals undergoing small deformations, International Journal of Solids and Structures, 39, 26, 6281-6309 
4. Clark W.A.T., Wagoner R.H., Shen Z.Y., Lee T.C., Robertson I.M., Birnbaum H.K., 1992, On the criteria for slip transmission across interfaces in polycrystals, Scripta Metallurgica et Materialia, 26, 2, 203-206

5. Coleman B.D., Noll W., 1963, The thermodynamics of elastic materials with heat conduction and viscosity, Archive for Rational Mechanics and Analysis, 13, 3, 167-178

6. Forest S., 2009, Micromorphic approach for gradient elasticity, viscoplasticity, and damage, Journal of Engineering Mechanics - ASCE, 135, 3, 117-131

7. Fredriksson P., Gudmundson P., 2007, Competition between interface and bulk dominated plastic deformation in strain gradient plasticity, Modelling and Simulation in Materials Science and Engineering, 15, 1, S61-S69

8. Gudmundson P., 2004, A unified treatment of strain gradient plasticity, Journal of the Mechanics and Physics of Solids, 52, 6, 1379-1406

9. Gurtin M.E., 2008, A theory of grain boundaries that accounts automatically for grain misorientation and grain-boundary orientation, Journal of the Mechanics and Physics of Solids, 56, 2, 640-662

10. Hirth J.P., Lothe J., 1982, Theory of Dislocations, Elsevier

11. Lee T.C., Robertson I.M., Birnbaum H.K., 1989, Prediction of slip transfer mechanisms across grain-boundaries, Scripta Metallurgica, 23, 5, 799-803

12. Nix W.D., Gao H.J., 1998, Indentation size effects in crystalline materials: A law for strain gradient plasticity, Journal of the Mechanics and Physics of Solids, 46, 3, 411-425

13. Ohmura T., Minor A.M., Stach E.A., Morris J.W., 2004, Dislocation-grain boundary interactions in martensitic steel observed through in situ nanoindentation in a transmission electron microscope, Journal of Materials Research, 19, 12, 3626-3632

14. Soer W.A., Aifantis K.E., De Hosson J.T.M., 2005, Incipient plasticity during nanoindentation at grain boundaries in body-centered cubic metals, Acta Materialia, 53, 17, 4665-4676

15. Song Y., Voyiadjis G.Z., 2018, Small scale volume formulation based on coupled thermo-mechanical gradient enhanced plasticity theory, International Journal of Solids and Structures, 134, March 2018, 195-215

16. Sun S., AdAms B.L., KInG W.E., 2000, Observations of lattice curvature near the interface of a deformed aluminium bicrystal, Philosophical Magazine a-Physics of Condensed Matter Structure Defects and Mechanical Properties, 80, 1, 9-25

17. VocE E., 1955, A practical strain-hardening function, Metallurgica, 51219-51226

18. Voyiadjis G.Z., Faghini D., Zhang Y.D., 2014, A theory for grain boundaries with strain-gradient plasticity, International Journal of Solids and Structures, 51, 10, 1872-1889

19. Voyiadjis G.Z., Song Y., 2017, Effect of passivation on higher order gradient plasticity models for non-proportional loading: energetic and dissipative gradient components, Philosophical Magazine, 97, 5, 318-345

20. Voyiadjis G.Z., Song Y., PARK T., 2017, Higher-order thermomechanical gradient plasticity model with energetic and dissipative components, Journal of Engineering Materials and Technology - Transactions of the ASME, 139, 2 